\title{
ON DIRECT PRODUCTS OF GENERALIZED SOLVABLE GROUPS
}

\author{
BY RICHARD E. PHILLIPS
}

\author{
Communicated by W. Feit, May 26, 1967
}

Let $G_{\alpha}(\alpha \in \Gamma)$ be a set of groups. The direct product $\prod\left\{G_{\alpha} \mid \alpha \in \Gamma\right\}$ is the set of all functions $f$ on $\Gamma$ such that $f(\alpha) \in G_{\alpha}$ for all $\alpha \in \Gamma$, with multiplication of functions defined componentwise. The direct sum $\sum\left\{G_{\alpha} \mid \alpha \in \Gamma\right\}$ is the subgroup of $\prod\left\{G_{\alpha} \mid \alpha \in \Gamma\right\}$ consisting of all functions $f$ with finite support.

A collection $B$ of groups is called a class of groups if $E \in B$, and isomorphic images of $B$ groups are $B$ groups. We use the following notation of $\mathrm{P}$. Hall [1]. If $\beta$ is a class of groups, $S(B), Q(B), D S(B)$, $D P(B)$ denote respectively the classes of groups which are subgroups, quotient groups, direct sums and direct products of $B$ groups.

The following theorem was proved by Merzulakov in [2].

THEOREM 1. If $\circledast$ is a class of groups satisfying

(a) $S(B)=\beta$,

(b) $Q(B)=B$,

(c) $G$ is a finite $B$ group if and only if $G$ is nilpotent, then $D P(B) \neq B$.

In this paper, a similar theorem is obtained for generalized solvable groups. Before stating these results, we need several definitions.

Definition 1. Let $G$ be a group, $x \in G, g \in G$. Define $[g, 0 x]=g$, and inductively $[g, n x]=[[g,(n-1) x], x]$ for each positive integer $n$. $x$ is called a left $G$ Engel element if for each $g \in G$ there exists an integer $n=n(g)$ such that $[g, n x]=e$.

The Hirsch-Plotkin radical of a group $G$ is the maximum normal locally nilpotent subgroup of $G$. We denote the Hirsch-Plotkin radical of $G$ by $\phi_{1}(G)$.

Definition 2. Let $G$ be a group and $\phi_{0}(G)=E$. If $\alpha$ is not a limit ordinal, define $\phi_{\alpha}(G)$ by $\phi_{\alpha}(G) / \phi_{\alpha-1}(G)=\phi_{1}\left(G / \phi_{\alpha-1}(G)\right)$. If $\alpha$ is a limit ordinal, define $\phi_{\alpha}(G)$ by $\phi_{\alpha}(G)=\bigcup\left\{\phi_{\beta} \mid \beta<\alpha\right\}$. If for some ordinal $\sigma$, $\phi_{\sigma}(G)=G, G$ is called an $L N$-radical group.

In the following, $\mathscr{L}$ will denote the class of $L N$-radical groups. If $G \in \mathcal{L}$, and $\sigma$ is the least ordinal for which $\phi_{\sigma}(G)=G, \sigma$ is called the radical class of $G$. It is well known that $S(\mathfrak{L})=\mathfrak{L}, Q(\mathfrak{L})=\mathfrak{L}$, and that every solvable group is in $\mathscr{L}$ [3]. It is easily shown that if $n$ is a positive integer, there exist finite solvable groups of radical class $n$ $[4$, p. 220].

We need the following theorem of Plotkin [3]. 
Theorem 2. If $G \in \mathcal{L}$, then the set of left Engel elements of $G$ is a subgroup, and this subgroup coincides with the Hirsch-Plotkin radical of $G$.

In the remainder of this paper, $J$ will denote the set of nonnegative in tegers.

Theorem 3. Let $n \in J$ and $G_{n} \in \mathscr{L}$ have radical class $n$. Then $G$ $=\prod\left\{G_{n} \mid n \in J\right\} \notin \&$.

Proof. Let $R_{k}=\prod\left\{\phi_{k}\left(G_{n}\right) \mid n \in J\right\}$ and $R=U\left\{R_{k} \mid k \in J\right\}$. Then $R \triangleleft G$ and $R \neq G$. We show that $\phi_{1}(G / R)=E$.

Suppose to the contrary that $\phi_{1}(G / R) \neq E$ and let $y R \in \phi_{1}(G / R)$ with $y \notin R$. Then $y R$ is a left $G / R$ Engel element. Thus for each $x \in G \backslash R$, there exists a positive integer $n=n(x)$ such that $[x, n y] \in R$. Hence for each $x \in G \backslash R$, there exist nonnegative integers $n=n(x)$ and $k=k(x)$ such that $[x, n y] \in R_{k}$.

We now construct an $x \in G$ for which the above assertions do not hold. Since $y \notin R$, there exists $i_{1} \in J$ such that $y\left(i_{1}\right) \notin \phi_{1}\left(G_{i_{1}}\right)$. By Theorem 2, $y\left(i_{1}\right)$ is not a left $G_{i_{1}}$ Engel element. Hence there exists $x_{i_{1}} \in G_{i_{1}}$ such that $\left[x_{i_{1}}, s y\left(i_{1}\right)\right] \notin \phi_{0}\left(G_{i_{1}}\right)=E$ for all $s \in J$.

Suppose nonnegative integers $i_{1}<i_{2}<\cdots<i_{r}$ and elements $x_{i_{j}}$ $\in G_{i_{j}}(1 \leqq j \leqq r)$ have been found so that for $1 \leqq j \leqq r,\left[x_{i_{j}}, s y\left(i_{j}\right)\right]$ $\notin \phi_{j-1}\left(G_{i_{j}}\right)$ for all $s \in J$. Since $y \notin R$, there exists an integer $i_{r+1}>i_{r}$ such that $y\left(i_{r+1}\right) \notin \phi_{r+1}\left(G_{i_{r+1}}\right)$. Thus, by Theorem $2 y\left(i_{r+1}\right) \phi_{r}\left(G_{i_{r+1}}\right)$ is not a left $G_{i_{r+1}} / \phi_{r}\left(G_{i_{r+1}}\right)$ Engel element. Hence there exists $x_{i_{r+1}}$ $\in G_{i_{r+1}}$ such that $\left[x_{i_{r+1}}, s y\left(i_{r+1}\right)\right] \notin \phi_{r}\left(G_{i_{r+1}}\right)$ for all $s \in J$.

Let $I=\left\{i_{1}, i_{2}, \cdots, i_{r}, \cdots\right\}$. Define $x \in G$ as follows: $x(\eta)=x_{\eta}$ if $\eta \in I$ and $x(\eta)=e$ otherwise. Let $k \in J$. Then $[x, s y] \notin R_{k}$ for all $s \in J$. This is contrary to the first paragraph of this proof.

THEOREM 4. Let $\otimes$ be a class of groups such that

(a) $B \subset \mathbb{L}$,

(b) every finite solvable group is contained in $B$.

Then $D P(B) \neq B$.

Proof. The proof follows from Theorem 3 and the existence of finite solvable groups of radical class $n$ for each $n \in J$.

The direct product $\prod\left\{G_{\alpha} \mid \alpha \in \Gamma\right\}$ is called a direct power of $H$ if each $G_{\alpha}$ is isomorphic to $H$. If $B$ is a class of groups, $d p(B)$ will denote the class of groups which are direct powers of $B$ groups.

In the next theorem, $\subseteq$ will denote the class of solvable groups.

THEOREM 5. If $B$ is a class of groups such that

(a) $B C \&$,

(b) $D S(s) \subset ৫$,

Then $d p(B) \neq B$. 
Proof. Let $G=\sum\left\{G_{n} \mid n \in J\right\}$ where $G_{n}$ is solvable of radical class $n$. Then $G \in \mathbb{B}$ and has radical class $\omega$. Let $H=\prod\left\{H_{k} \mid k \in J, H_{k} \simeq G\right\}$. $H$ has a subgroup satisfying the hypothesis of Theorem 3 . Hence $H \notin \mathcal{L}$. Consequently, $H \notin ß$.

Classes of groups satisfying the conditions of Theorems 4 and 5 include the classes $S N^{*}, S I^{*}$, subsolvable and polycyclic.

\section{BIBLIOGRAPHY}

1. P. Hall, On non-strictly simple groups, Proc. Cambridge Philos. Soc. 59 (1963), 531-553.

2. J. I. Merzulakov, On the theory of generalized solvable and nilpotent groups, Algebra i Logika Sem. 2 (1963), 29-36. (Russian)

3. B. I. Plotkin, Radical groups, Amer. Math. Soc. Transl. (2) 17 (1961), 9-28.

4. W. R. Scott, Group theory, Prentice Hall, Englewood Cliffs, N. J., 1965.

UNIVERSITY OF KANSAS

\section{ALGEBRAIZATION OF ITERATED INTEGRATION ALONG PATHS ${ }^{1}$}

BY KUO-TSAI CHEN

Communicated by Saunders Mac Lane June 12, 1967

If $\Omega$ is the vector space of $C^{\infty} 1$-forms on a $C^{\infty}$ manifold $M$, then iterated integrals along a piecewise smooth path $\alpha:[0, l] \rightarrow M$ can be inductively defined as below:

For $r \geqq 2$ and $w_{1}, w_{2}, \cdots, \in \Omega$,

$$
\int_{\alpha} w_{1} \cdots w_{r}=\int_{0}^{l}\left(\int_{\alpha^{t}} w_{1} \cdots w_{r-1}\right) w_{r}(\alpha(t), \dot{\alpha}(t)) d t
$$

where $\alpha^{t}=\alpha \mid[0, t]$. (See [3].)

This note is based on the following algebraic properties of the iterated integration:

(a) $\left(\int_{\alpha} w_{1} \cdots w_{r}\right)\left(\int_{\alpha} w_{r+1} \cdots w_{r+s}\right)=\sum \int_{\alpha} w_{\sigma(1)} \cdots w_{\sigma(r+s)}$ summing over all $(r, s)$-shuffles, i.e. those permutations $\sigma$ of $\{1, \cdots, r+s\}$ with $\sigma^{-1}(1)<\cdots<\sigma^{-1}(r), \sigma^{-1}(r+1)<\cdots<\sigma^{-1}(r+s)$.

(b) If $p=\alpha(0)$ and if $f$ is any $C^{\infty}$ function on $M$, then

$$
\int_{\alpha} f w=\int_{\alpha}(d f) w+f(p) \int_{\alpha} w .
$$

1 The work has been partially supported by the National Science Foundation under Grant NSF-GP-5423. 